\title{
NOTES ON A SERIES OF SEVENTY-FIVE CASES OF GUNSHOT WOUNDS OF THE CHEST.
}

\author{
By Captain J. L. MENZIES, R.A.M.C.
}

ONE of the most striking features of a modern military hospital is the large number of cases of penetrating gunshot wounds of the chest admitted with comparatively trivial symptoms. The immediate results following such injuries are often marked and severe, but those patients who survive and are sent to a base hospital are often surprisingly well when first admitted. The experience of military surgeons in the present campaign serves to confirm the observations made in the South African War, that the modern highvelocity, hard-jacketed rifle bullet can pass through almost any part of the lung, and sometimes probably through the heart, without death of the patient resulting. -

It is impossible to calculate with any degree of accuracy how many cases of gunshot wounds of the chest die on the field of battle from injury to the heart or.large vessels, but it would seem that even extensive wounds of the lung and the chest wall may be recovered from. The actual site of penetration of the chest is undoubtedly of importance on the field of battle, as many with injuries to the root of the lungs and large vessels, die soon after injury; but in those who recover sufficiently to be removed to a base hospital, injuries are met with in every conceivable portion of the chest, and recovery is little affected by the site of the wound.

A very noticeable feature in a series of cases of gunshot wounds of the chest is the rapid recovery where there is no destruction of the chest wall, compared with the high mortality in cases with an open wound on arrival at the base. Cases where a wound in the chest opened into the pleural cavity, with communication between that cavity and the outer air, were almost uniformly fatal.

At the 21st General Hospital at Ras-el-Tin, Alexandria, 75 cases of gunshot wounds of the chest were admitted between July 1 and Sept. 31, 1915. By the courtesy of Captains Blacker, Wareham, and Whittaker, in charge of surgical wards, I had the opportunity of examining all these cases. This hospital serves as one of the large base hospitals for operations in Gallipoli. Cases are brought by hospital ship from the Peninsula to Alexandria, and the average time in this series of cases between injury and admission to hospital was five to six days, most of which time was spent on a well-equipped hospital ship.

When the chest wall and the contained viscera are penetrated by a projectile, certain marked symptoms present themselves. These symptoms, in their order of frequency, are pain, shock, dyspnoea, and hæmoptysis, all of 
which occur at the time of the injury or soon after. They are referable in the first place to damage to lung tissue, and secondly to the resulting escape of blood or air into the pleural cavity, caused by damage to lung or blood-vessels.

Pain is present to a greater or less degree in all cases. It is rarely very severe, and calls for little comment.

Shock may be severe, at times resulting in collapse and loss of consciousness. A few cases were able to walk after receiving a penetrating wound of the chest, but these were exceptional.

Dyspnœe is almost always present at the time of the injury, and is generally characteristic. The distress in breathing in most cases is so great that the patient appears to be choking, and is continually fighting for breath. It is most noticeable in cases where there is a large sudden effusion of blood or escape of air into the pleural cavity: In most cases the dyspnoea improves after twelve to twenty-four hours, when the lungs become accustomed to the unusual conditions. Where effusion of blood in the pleural cavity is increas-

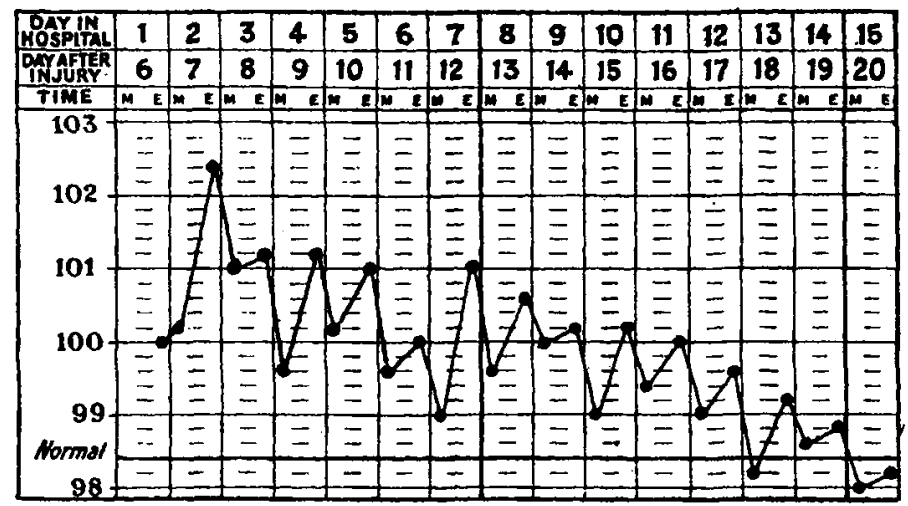

Fig. 253.-Temperature Chart in a Case of Sterile Hamothorax.

ing, the dyspnoea generally increases proportionately. Among 75 cases, only 12 gave no history of dyspnoea.

Hæmoptysis occurs in the majority of cases. It occurs either immediately after the injury, or after a delay of some hours. It is seldom very. severe, and generally lasts two to three days, but there may be clots for a week or more. Of the 75 cases, 27 had no hæmoptysis at any time; in 40 cases it came on immediately after injury; in 8 it was delayed from one to twelve hours. The actual amount of blood coughed up is difficult to estimate under the best circumstances, and the various estimates of patients are certainly very erroneous. The amount varies from 'clots' to a pint.

The temperature is said to be elevated in all cases after chest injury.

On admission to the base hospital, the earlier symptoms have disappeared, and the symptoms which are now present depend on the existing conditionwhether therc is effusion or not, and whether the effusion is -septic or sterile. The most prominent symptoms are dyspnca and pyrexia. Most cases show signs of effusion of blood into the pleural cavity, and a minority signs of blood 
and air. Bronchitis is present in nearly all cases. Surgical emphysema of the chest wall occurs in a few.

The dyspnoea is generally proportionate to the amount of effusion and consequent displacement of the mediastinum. Occasionally cases occur with a large effusion and comparatively little distress in breathing.

Rise of temperature is said to occur always after effusion of blood into the pleural cavity. On admission to hospital the temperature in the majority of cases was elevated to $100^{\circ}$ or $101^{\circ}$, and even to $103^{\circ}$ or $104^{\circ}$, and reached normal in about ten to fourteen days in sterile cases; but in 9 cases the temperature was normal on admission, and remained so. Fig. 253 shows a typical temperature chart from a case of sterile hæmothorax.

Hæmothorax was present in varying degree in all except 5 cases. In 49 the effusion was sterile, in 10 pus was mixed with blood, and in 11 the blood was found to be infected on bacteriological examination.

Pneumothorax was present in 14 cases. In 8 the pneumothorax communicated with the outer air through an opening in the pleura, and in 6 the pneumothorax was closed. The pneumothorax was never simple, but always associated either with blood alone or with blood and pus.

Bronchitis in varying degree was present in most cases.

Surgical emphysema was present in 12 cases. It was never very extensive, and required no special treatment.

Of the 75 cases admitted to hospital, 56 were wounded by rifle bullets, and 19 by shrapnel or bombs. The latter wounds were more severe as a rule. A large proportion of projectiles were retained in the chest. This was the case on admission in 35 instances-26 bullets and 9 pieces of shrapnel : 4 rifle and 4 shrapnel bullets were extracted from the chest wall or the pleural cavity, and 27 patients were discharged from hospital with the projectile still retained and apparently causing no symptoms. The presence or absence of the missile seems to make little difference to recovery.

Of the entire series of 75 cases, only 5 had no effusion into the pleural cavity.

The cases could be divided into two distinct classes :

$A$. Those with penetrating wounds which were healed or healing at the time of admission into the base hospital. This class may be subdivided as follows: (1) Those with hæmothorax; (a) Sterile, (b) Infected-(i) from without, (ii) from within; (2) Those without hæmothorax.

$B$. Those with penetrating wounds opening into the pleural cavity at the time of admission. In this class all had empyema on the side of the injury.

The first class contains the larger proportion of the cases, and they are much more favourable for treatment. There were 67 cases in this class: 66 recovered, and 1 died of sepsis. The large majority had a larger or smaller effusion of blood into the pleural cavity. As stated above, only 5 cases with definitely penetrating wounds of the lung showed no evidence of effusion. The great majority of the effusions were bacteriologically sterile, and consisted generally of brownish liquid blood. In cases which were aspirated, this blood generally clotted in the vessel after being drawn off.

In the second class the wounds were much more severe, and there was 
great destruction of the chest wall. The mortality was very high. All the cases were very ill and septic on admission; all had pyohæmopneumothorax, and drainage of the empyema did not give much relief. There were 8 of these cases, with 7 deaths. Only one case recovered where the pleura was perforated at the time of admission, and where air entered the pleural cavity with respiration. The period that elapsed between the time of injury and death varied from ten to twenty-one days. Post-mortems showed in all cases extensive laceration of the lung, generally with a lung abscess spreading from the bullet track. Abscess of the mediastinum occurred in two cases.

In the foregoing series of 75 cases, 67 recovered and 8 died. Sepsis was the only cause of death. All the cases had been moved from the firing line to the base by hospital ship immediately after the injury, and no untoward results from early removal were noted. Of the 5 cases with no effusion into the pleural cavity, all recovered completely without special treatment. In 70 cases effusion of blood occurred; in $\mathbf{4 9}$ of these the effused blood was bacteriologically sterile, and all recovered; in 11 there was early or mild infection, and all recovered; the remaining 10 had a considerable amount of pus in the pleural cavity, with septic absorption, and 8 of these died of sepsis.

Treatment.- All cases of perforating wounds of the chest must be carefully watched from the time of injury. The immediate symptoms generally improve when the patient is kept quiet. Hæmoptysis ceases spontaneously in nearly all cases. At the time of injury morphia may be given, and any bleeding from intercostal arteries should be arrested; but as a rule no further treatment is necessary beyond keeping the patient quiet.

On admission to hospital, when there is no effusion into the pleural cavity no special treatment is necessary, and recovery is rapid.

When effusion is present, the chest should be explored at any early date, and the fluid examined bacteriologically. When the fluid is found to be sterile, it may or may not be removed : in cases where the effusion is large, with marked dyspncea and much displacement of the mediastinum, it is always advisable to aspirate the fluid; when the effusion is not large, it may be aspirated or left alone. In cases where it is left alone, recovery is rather slower. Of 49 cases of sterile hæmothorax, 16 were aspirated and 33 were not. All recovered, and all were discharged in four to six weeks; in both series there remained some impairment of note over the base on the side of the effusion.

When a hremothorax is found to be infected, it should be aspirated or drained early. It was found in this series that resection of a portion of rib, and drainage by tube. gave most satisfactory results. Of 5 cases where fluid was aspirated, resection of a rib was performed later in 4 . Of 11 cases of infected hæmothorax, 10 had a portion of rib resected; all recovered.

When definite pus is present, the pleural cavity should be drained at the earliest possible time by the resection of a portion of a rib and the insertion of a large drainage tube. Where a large empyema cavity exists, weak iodine body baths were found beneficial; or gentle lavage of the pleural cavity through a tube without pressure may be employed. There were 10 cases of 


\section{GUNSHOT WOUNDS OF THE CHEST}

pyothorax : 8 were associated with open wounds, with 7 deaths; 2 with closed wounds, with 1 death.

The majority of the cases occasioned no anxiety from the time of admission; their recovery was uninterrupted, although signs of fluid were present for a long time after the patients were apparently well. It was found beneficial to get them up early, even before the temperature was quite normal, and to practise gentle exercises and breathing exercises.

It is impossible at present to say what will be the after-history of these cases, and whether they will be able for the most part to perform their ordinary duties. Probably in all cases the pleura must be much thickened, and there will be numerous adhesions binding down the lung, and impairment of its movement, resulting in shortness of breath and fatigue on exertion. It would be most useful to follow the after-history, but the difficulties of doing so are very considerable.

I have to thank Lieutenant-Colonel Robinson, in charge of the 21st General Hospital, for permission to publish these cases.

\section{CASES PRESENTING UNUSUAL FEATURES.}

\section{I.-Three Cases of Penetration of the Chest over the Precordium.}

Case 1.-Private T. C. was hit by a rifle bullet on July 13 when standing in a trench. He had severe pain and much dyspnca, and hæmoptysis immediately after injury. He was admitted six days after being wounded, with severe dyspnoea and much cyanosis, temperature $102^{\circ}$, pulse 98, respiration 36. The bullet entered the left side of the chest one and a half inches above and three-quarters of an inch inside the nipple line. The wound of exit was in the tenth space in the mid-scapular line. Both wounds were healed on admission. There was extensive surgical emphysema over a large area of the chest around the entrance wound, extending from the clavicle to the sixth rib in front. The left side of the chest moved badly. In front there was a markedly hyper-resonant, almost tympanitic, note over the whole of the left side. Cardiac dullness was absent, and the apex beat was not palpable. On auscultation, the maximum intensity of the apex beat was in the sixth space half an inch external to the left margin of the sternum. Behind, there was marked dullness on the left side from the base to the spine of the scapula. There was a well-marked bruit d'airain, and there were other signs of pneumohæmothorax. The chest was explored, and a small quantity of dark brown liquid blood drawn off was found bacteriologically sterile. The temperature showed a daily excursion of two to three degrees, from $100^{\circ}$ to $103^{\circ}$; it gradually subsided, and reached normal seventeen days after admission. The surgical emphysema cleared up completely three days after admission, and the signs of pneumothorax cleared up within a week, leaving the signs of simple hæmothorax. The patient had no special treatment, and was discharged well within a month.

The features in this case were $(a)$ The track of the bullet; and $(b)$ The general appearance of sepsis in a case where examination of the effused blood showed the latter to be sterile.

Case 2.--Bandsman S. H. was carrying a stretcher on June 17, when he was hit by a rifle bullet. He walked five hundred yards after being hit, and said he felt very little discomfort until he began to feel weak. There was little bleeding from the wound at the time, no hæmoptysis, and little dyspncea. He was admitted on June 24, feeling very well, with little distress, and with normal temperature, pulse, and respiration. The bullet entered the fourth space just outside the right sternal 
line, at the junction of the cartilage with the sternum. The wound was healed on admission, and the bullet retained in the chest. He had signs of effusion at the left base. On June 27 he had severe pain in the left side, and was found to have a well-marked pericardial friction rub. The temperature was elevated to $101^{\circ}$, respiration 40, and pulse 103 . The chest was aspirated on June 30 , and a pint and a half of dark brown fluid blood was removed from the left pleural cavity, and was found to be sterile. There were signs of fluid in the pericardium, which was possibly serum, but it was not explored. $X$-ray examination showed the heart area greatly enlarged and increased in density. The patient was much relieved by tapping and the temperature came gradually to normal in three weeks, when signs of effusion in the pericardium had disappeared.

The interest of this case is concerned mainly with the track of the bullet, which must have pierced the pericardium, and possibly also the heart, causing no immediate symptoms, but the later development of a simple pericarditis, with complete recovery.

Case 3.-Private P. M. was hit by a rifle bullet on Sept. 1. He had hæmoptysis for four days and much dyspnœa, but no loss of consciousness. He was admitted on Sept. 8, and was not very ill. On admission, the temperature was $101^{\circ}$, pulse 98, and respiration 24. The wound of entry was half an inch above and two inches internal to the nipple line on the left side. The wound of exit behind was three inches from the spine, over the eighth rib. There were signs of a large hæmothorax on the left side, with considerable displacement of the heart to the right. There was no evidence of damage to heart or pericardium. The hamothorax was found to be sterile, but fluid was not aspirated. The temperature ran an irregular course, varying from $99^{\circ}$ to $101^{\circ}$, and then gradually to normal, in fifteen days. The patient was discharged after four weeks, with very much diminished evidence of fluid.

In this case the entrance of the bullet was over the cardiac area, but no serious results followed.

\section{II.-Two Cases of Penetration of Both Lungs and Mediastinum, with Double Hæmothorax.}

Case 4.-Private W. J. was hit by a rifle bullet on Aug. 31. He had hæmoptysis lasting a day and a half-about a cupful altogether. He also had well-marked dyspnœa. He was admitted to the base hospital on Sept. 9, with slight dyspnœa and little elevation of temperature. The bullet was found to have entered the fourth space on the right side, one inch internal to the mid-clavicular line. It passed through the lungs, and evidently through the posterior mediastinum, to emerge in the fifth intercostal space in the posterior axillary line on the left side. There were signs of a large effusion on the right side and a smaller one on the left, with some cardiac displacement to the left. The hæmothorax on both sides was sterile, and was not aspirated. Signs of effusion had decreased greatly on discharge.

Case 5.-Lance-Corpl. C. W. was hit by a rifle bullet on Sept. 8 while bending. He had much dyspnœea and hæmoptysis directly after the injury. He was admitted to the hospital on Sept. 14; his temperature was $101^{\circ}$, pulse 98, respiration 26 . The entrance wound was in the fourth intercostal space on the right side, one inch behind the posterior axillary line. The bullet passed through both lungs, to emerge through the left shoulder. There were signs of a large effusion in the right pleural cavity, and of a smaller effusion at the left base; both were sterile. Two pints of sterile blood were removed from the right pleural cavity on Sept. 15. The patient made an uninterrupted recovery, and was discharged within a month with few physical signs.

\section{III.-Large Hemothorax with relatively Few Symptome.}

Case 6.-Corpl. C. O. was wounded on July 13 by a rifle bullet while crouching in a trench. He had no hæmoptysis, and little distress in breathing. On admission, seven days after the injury; the patient was very comfortable, he had no dyspnœa, and pulse, temperature, and respiration were normal, and remained so. The bullet 
entered through the second intercostal space on the left side in the mid-clavicular line, and was retained, but could not be located by $x$ rays. There were signs of a large hæmothorax on the left side, with dullness extending almost to the apex behind, and to the fourth rib in front. The heart was considerably displaced to the right. The chest was explored, and the dark brown fluid blood withdrawn was found to be sterile. The chest was not aspirated, and the patient was discharged on Aug. 20 with physical signs of effusion very much diminished.

IV.-Three Cases of Infected Bmemothorax where Apparently Pure Blood withdrawn by the Hxploring Syringe was found to be Infected on Bacteriologioal Framination.

(There were 11 cases of infected hæmothorax; the most common infections were the pneumococcus and staphylococcus. All recovered.)

Case 7.-Private G. S. was hit by a rifle bullet on Aug. 18 while sleeping. At the time of injury he had much distress in breathing, and hæmoptysis which lasted for two days. He was admitted to hospital on Aug. 24, with temperature $101^{\circ}$, pulse 96, and respiration 32. The entrance wound of the bullet was on the right side in the anterior axillary line between the eighth and ninth ribs. The bullet was retained. There were signs of effusion on the right side, extending to the spine of the scapula behind. The heart was displaced to the left. On Sept. 3 his temperature rose to $104^{\circ}$, with pulse 106, and respiration 36, and the signs of fluid had increased. The chest was explored, and dark brown fluid blood was drawn off by the syringe. This was examined bacteriologically, and pneumococci were found to be present. A portion of the ninth rib was resected, about two pints of infected blood were evacuated, and the pleural cavity was drained by tube. The patient recovered rapidly, and the temperature became normal in about three weeks. On discharge, the wound was healed and the lung was expanding.

Case 8.-Private A. P. received a bullet wound in the chest on Aug. 9. He had hæmoptysis half an hour after the injury. Dyspnca was not marked at the time,

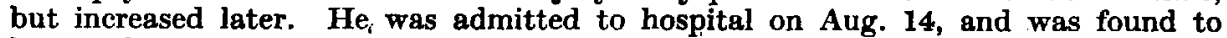
have a bullet wound in the fifth right intercostal space just behind the posterior axillary line. The fourth and fifth ribs were fractured at the site of injury. The bullet was retained. There were signs of effusion into the right pleural cavity, extending to the angle of the scapula behind. On Aug. 19 one pint of sterile blood was aspirated from the right pleural cavity. On Aug. 25 signs of hæmothorax had increased, and the temperature rose to $103^{\circ}$. He was again aspirated, and the fluid was bacteriologically examined, and found to contain pneumococei. On Aug. 26 a portion of the ninth rib was resected; and the pleural cavity was drained by tube. A deformed bullet, found free in the pleural cavity, was removed. The patient developed bronchopneumonia, which delayed his convalescence. He was discharged on Sept. 28 with the wound healed, and with signs of air entry into the base of the right lung.

Case 9.-Private F. G. received a bomb wound in the back on Aug. 6, while standing on the side of a trench. He fell into the trench, a distance of five feet, when he probably fractured a rib. He had a little hæmoptysis at times, and he coughed up blood for ten days. He had much dyspncea, and a great deal of pain on the right side. He was admitted to hospital on Aug. 13, with slight elevation of temperature and little dyspncea. He had a wound in the back on the right side, two inches from the spine, at the level of the eighth rib. This rib was fractured at its angle. On examination, he showed signs of effusion on the right side, with dullness from the base to the angle of the scapula. The apex beat was in the fourth space, half an inch outside the nipple line. On Aug. 18 his distress became greater, and he had much pain in the right side. The effusion was found to be increasing, and the dullness and absence of breath sounds extended to the apex. The fluid was aspirated, and three pints of dark fluid blood were drawn off. The blood clotted in the vessel. After aspiration the patient was much more comfortable, but was 
distressed again on the following day, and on the 20th the signs of fluid had again reached the apex, and the mediastinum was considerably displaced to the left. The apex beat was in the fifth space in the anterior axillary line. The chest was again aspirated, and two pints of blood were withdrawn, which was found on bacteriological examination to contain streptococci. A portion of the eighth rib was resected, and the chest drained. Subsequently the pleural cavity was washed daily by weak iodine solution through funnel and tube. The patient made an excellent recovery, although convalescence was delayed by an attack of dysentery. On discharge, on Oct. 1, the wound was healed, and a definite amount of air was entering the lung.

\section{V.-Pyothorax followed by Recovery.}

Case 10.-Private G. N. received a bullet wound in the back on Aug. 8. He had no hæmoptysis and no dyspncea. He was admitted to hospital on Aug. 13, with a temperature of $100^{\circ}$, pulse 106 , and respiration 30 . He had a bullet wound opposite the third lumbar vertebra one inch to the left of the spine, and no exit wound. There were signs of effusion in the left chest from the base to the 'spine of the scapula. The chest was explored, and a small amount of blood and pus withdrawn, which was found to contain streptococci. A part of the twelfth rib was excised, and one pint of blood and pus was evacuated from the pleural cavity. The bullet casing was removed from a pocket behind the eleventh rib. The cavity was drained by tube, and a tube was also inserted to drain a perinephric collection of pus. The patient had daily body baths of weak iodine. He made a complete recovery, and the wound was healed on discharge.

\section{VI.-Pyopneumothorax followed by Recovery.}

Case 11.-Private G. K. was hit in the back by a bullet on Aug. 19. He had much dyspnoea and hæmoptysis at the time of injury. He was admitted to hospital on Aug. 24 with a temperature of $101^{\circ}$, pulse 96 , and respiration 30 . There was a healed entrance wound in the eighth space behind, in line with the angle of the scapula. The exit wound was in the first intercostal space, one inch outside the border of the manubrium sterni. The anterior wound was gaping, exposing the pleura, and in the centre of the latter there was a small pinhole puncture, with air entry during respiration, and some oozing of pus. There was surgical emphysema for a radius of three inches around the wound. There were signs of a pneumohæmothorax, which was evidently infected. A portion of the ninth rib was resected in the posterior axillary line, and one pint of blood and pus evacuated. The upper wound was also explored, and half a pint of pure pus evacuated, evidently a separate loculus shut off from the lower collection. Both wounds were drained by tube. On discharge, both wounds had healed, and there were signs of lung expansion.

\section{Two Cases of Pyohremothorax with Open Pneumothorax. Post-mortem Findings.}

(Altogether there were 8 cases, with $\gamma$ deaths.)

Case 12.-Private G. O. was digging on Aug. 7 when he was hit by a sniper. He gasped for breath. and coughed up a little blood immediately after the injury. He was admitted on Aug. 14, very ill, cyanosed, and gasping for breath, with a temperature of $102^{\circ}$, pulse 108 , and respiration 36 . There was a bullet wound through the middle of the right clavicle, with fracture of the clavicle and an open wound into the pleural cavity. Air entered the pleural cavity with respiration. The exit wound was in the ninth space in the mid-scapular line. The intercostal spaces on the right side were obliterated, there was marked hyper-resonance over the whole of the right chest, absence of breath sounds over the lower lobe, and there were faint cavernous breath sounds over the upper lobe. Posteriorly there was dullness from the base to the angle of the scapula. Metallic tinkling was well marked, and the coin sound was elicited. There were coarse râles and rhonchi over both lungs, and a friction rub from the second to the fourth rib in front. The patient died twenty-four hours after admission. 


\section{GUNSHOT WOUNDS OF THE CHEST}

Post-mortem Examination.-The bullet track passed through the clavicle downwards and backwards, fracturing the second to the tenth ribs, and emerging in the ninth space in line with the angle of the scapula. The fractures of the clavicle and ribs were comminuted, with extensive laceration of the parietal pleura by sharp spicules of bone. There was wide laceration of the posterior parts of the right upper and lower lobes in the line of the fractured ribs, and complete collapse of the right lung. The pleural cavity contained air, and there were about two pints of bloodstained pus. There were also a few patches of bronchopneumonia of the left lung, with partial collapse.

Case 13.-Private J. S. received a bomb wound of the chest on Aug. 2. He had much dyspncea, and coughed up about a pint of blood at the time of injury and for two hours afterwards. He was admitted on Aug. 13, with much distress in breathing, and seemed very ill. The temperature was $100 \cdot 8^{\circ}$, pulse 136, respiration 38. He had a perforating wound on the right side of the chest communicating with the pleural cavity. The bullet entered between the fifth and sixth ribs in the midaxillary line, fracturing the ribs. The projectile was retained. Examination showed signs of effusion of blood and air into the pleural cavity. The wound was opened up, a large collection of blood and pus evacuated, and a drainage tube inserted. The draining of the pyothorax gave little relief; and the patient developed pneumonia on the left side, and died on Aug. 20.

Post-mortem Examination.-The projectile fractured the fifth and sixth ribs in the right mid-axillary line, passed to the left upwards and forwards through the right lung, and finished in the mediastinum behind the upper part of the manubrium sterni. A fragment of bomb casing, $1 \mathrm{~cm}$. in diameter, was found in a large abscess cavity just behind the sternum, with some erosion of the bone. There was a wide track full of pus through the lung, with signs of purulent bronchitis and peribronchial pneumonia in the surrounding lung tissue, and also bronchopneumonia of the left lung. 